\title{
Percepções dos estudantes da área de saúde sobre a obesidade
}

\section{Perceptions of health students on obesity}

\author{
Juliana Kessar Cordoni¹, Vanessa Keiko Rossaka¹, Lígia de Fátima Nóbrega Reato²
}

${ }^{1}$ Instituto de Hebiatria, Departamento de Pediatria, Faculdade de Medicina do ABC (FMABC) - Santo André (SP), Brasil.

${ }^{2}$ Disciplina de Hebiatria, Departamento de Pediatria, FMABC - Santo André (SP), Brasil.

DOI: http://dx.doi.org/10.7322/abcshs.v39i3.651

\section{RESUMO}

Introdução: A incidência de sobrepeso e obesidade, assim como agravos e comorbidades relacionados, tem se destacado como um dos principais problemas de saúde pública da atualidade. Isso requer o envolvimento de diversos profissionais, em uma conjunção multidisciplinar, para a prevenção, promoção e recuperação da saúde física e psicossocial de pessoas com sobrepeso ou obesidade. Objetivo: Identificar a percepção de estudantes da área de saúde sobre a obesidade, a fim de contribuir com questionamentos e reflexões com relação à formação e atuação deles no tratamento de pessoas com sobrepeso/obesidade. Métodos: Esta pesquisa foi realizada por meio da apresentação de figuras, seguida da aplicação de um questionário dirigido a universitários da área de saúde; e posterior análise qualitativa e quantitativa das respostas. Resultados: Os entrevistados reconheceram a importância do tratamento da obesidade, assim como a necessidade de preocupação com aspectos da saúde; no entanto, os resultados indicam a existência de rejeição e crítica quanto à obesidade na maioria dos entrevistados. Conclusão Os resultados são um alerta de que a questão da obesidade precisa ser mais bem abordada nos cursos de formação da área da saúde, para reduzir os efeitos negativos dessa rejeição/crítica no decorrer do tratamento de tais pacientes. Considerando-se o impacto de aspectos psicológicos e emocionais dos profissionais envolvidos no tratamento e a escassa produção técnica-científica sobre o tema, sugere-se a produção de outras pesquisas, assim como deve-se intensificar a abordagem e sensibilização dos estudantes quanto à questão da obesidade como um distúrbio alimentar de relevância pública.

Palavras-chave: obesidade; percepção; saúde; estudantes.

\begin{abstract}
Introduction: The incidence of overweight and obesity, as well as the related complications and comorbidities, has emerged as a current major public health issue. It requires the involvement of various professionals in a multidisciplinary combination for the prevention, promotion, and recovery of physical and psychosocial health of overweight or obese people. Objective: To identify the perceptions of health students on obesity, in order to contribute with questions and reflections about their formation and performance in the treatment of overweight/ obesity. Methods: This survey was carried out by presenting pictures, followed by the use of a questionnaire addressed to students from health university courses, and subsequent qualitative and quantitative analyses of the answers. Results: Respondents recognized the importance of treating obesity, as well as the need for concern on health aspects; however, results indicate the existence of rejection and criticism about obesity in most respondents. Conclusion: The results are a warning that the obesity issue needs to be better addressed in training courses in the health area in order to reduce negative effects of rejection/criticism during the treatment of these patients. Considering the impact of psychological and emotional aspects of the professionals involved in the treatment and scarce technical-scientific literature on the topic, it is suggested the production of other studies as well as improvement of approach and awareness of the students on the obesity issue as an eating disorder of public relevance.
\end{abstract}

Keywords: obesity; perception; health; students.

Recebido em: 18/03/2014

Revisado em: 28/08/2014

Aprovado em: 12/09/2014

Autor para correspondência: Juliana Kessar Cordoni - Avenida Príncipe de Gales, 821 - Príncipe de Gales - CEP: 09060-650 - Santo André (SP), Brasil E-mail: julianakc@hotmail.com

Conflito de interesses: nada a declarar. 


\section{INTRODUÇÃO}

A incidência de sobrepeso e obesidade, assim como os agravos e as comorbidades relacionados atinge, atualmente, um grau epidêmico. Em razão desse crescimento, ela tem sido considerada como um problema de saúde pública ${ }^{1}$, um contexto que favorece o aumento de questionamentos e pesquisas a níveis nacional e mundial.

A obesidade é considerada uma doença crônica não transmissí$\mathrm{vel}^{2}$, definida pelo acúmulo de gordura corporal que provoca alterações no corpo humano e com origem em causas multifatoriais, gerando impactos em diversas condições da vida humana, e, mais amplamente, na sociedade.

Essa condição requer o debruçamento de diversas áreas da saúde e coletividade, já que sua etimologia abrange fatores biopsicossociais, e a prevenção, promoção e recuperação da saúde física e psicossocial de pessoas com sobrepeso/obesidade prescindem da atuação de diversos profissionais, em uma conjunção multidisciplinar ${ }^{3,4}$.

Pode estar associada a causas de ordem fisiológica, como fatores genéticos, endócrinos, entre outros, ou sociais e psíquicos. É importante destacar o impacto de mudanças recentes nos hábitos de vida, principalmente pela expansão de alimentações ocidentalizadas, incluindo fast-food e afins, e o aumento do sedentarismo e diminuição progressiva da atividade física, intensificados pelos avanços tecnológicos ${ }^{5,6}$.

A incidência dos casos de sobrepeso/obesidade no Brasil aumentou, particularmente, no final do século 20. Dados estatísticos de 2006 demonstram que 8,9\% dos homens e 13,1\% das mulheres apresentavam sobrepeso. Os mesmos indicadores evidenciaram aumento de peso exacerbado em crianças e adolescentes. Nos Estados Unidos, os índices são superiores aos do Brasil, mas a estimativa do aumento de peso vem se mantendo nos patamares constatados por pesquisas ${ }^{7}$.

Segundo dados da Pesquisa de Orçamentos Familiares (POF) do Instituto Brasileiro de Geografia e Estatística (IBGE) ${ }^{8}$, 10,5 milhões de brasileiros com 20 anos ou mais são obesos na proporção apontada. Tal fato torna a obesidade a terceira doença nutricional no Brasil.

Como fator psicossocial pode-se destacar a influência midiática, principalmente pela sua participação na construção de modelos sociais e padrões de beleza. Os meios de comunicação em massa exploram a alimentação como consumo, ao mesmo tempo em que instituem um padrão estético corporal ideal ${ }^{9}$. Apresenta-se contraditoriamente, pois transmite mensagens que induzem à aquisição e ao consumo de alimentos que fornecem muitas calorias ao corpo, tais como lanches e produtos industrializados, mas, por outro lado, fixa-se um padrão estético calcado no corpo ideal, esquálido e musculoso, como símbolo de juventude e saúde ${ }^{10}$.

Pesquisas indicam que pessoas com obesidade são, frequentemente, estigmatizadas e vítimas de preconceito e discriminação, dificultando a aceitação pelo grupo social, podendo levar ao afastamento das atividades sociais. Nos Estados Unidos, por exemplo, a prevalência da discriminação de peso aumentou em $66 \%$ na última década, inclusive em contextos como ambientes de trabalho, centros de saúde e instituições de ensino ${ }^{11}$.

Observa-se menor aceitação de obesos em faculdades e ambientes profissionais. Alguns estudos demonstram que eles são menos aceitos nas faculdades e recebem menos bolsas de estudo do que os indivíduos que mantêm o peso controlado ${ }^{12}$. Outras investigações indicam a marginalização do obeso, em função de sua aparência, que se desvia dos modelos estabelecidos pela sociedade, na esfera do trabalho ou mesmo no campo afetivo ${ }^{13}$.

Em alguns casos, tais indivíduos podem ser considerados preguiçosos, desmotivados, indisciplinados, menos competentes e desleixados ${ }^{11}$, facilitando o estigma, a injustiça social e o preconceito. Em contrapartida, estas pessoas sentem-se descontentes, inferiores, com dificuldade de adaptação ao ambiente, além de apresentarem sentimentos de inadequação e depreciação diante dos julgamentos externos negativos ${ }^{6}$.

O impacto da obesidade na vida do sujeito pode comprometer a execução de atividades rotineiras, como higiene corporal, e domésticas, as quais podem representar grandes obstáculos ${ }^{14}$. A recuperação da qualidade de vida e da autoestima é fator preponderante para que o paciente possa se reintegrar em seu plano social.

Pesquisas apontam ainda que a obesidade nem sempre é entendida como uma enfermidade, mesmo entre os profissionais e estudantes da área da saúde, sendo que é frequentemente considerada como responsabilidade do paciente ${ }^{11}$. Isso é diferente de quando são tratados casos de anorexia, que causam, em geral, comoção e empatia diante da abstinência ou suspensão da alimentação por parte das equipes multidisciplinares e da sociedade em geral ${ }^{15}$ Embora tanto a anorexia quanto a obesidade sejam doenças associadas a distúrbios alimentares, a obesidade ainda é compreendida como uma responsabilidade do sujeito ${ }^{15}$.

Pelo fato dela ser uma doença que afeta o homem em múltiplos aspectos - físico, psíquico e social - e, por consequência, o corpo na sua totalidade ${ }^{15,16}$, os profissionais da área de saúde precisam compreender aspectos multifatoriais do indivíduo, a fim de viabilizar um tratamento adequado, global e satisfatório para quem está com sobrepeso/obesidade.

A partir da premissa de que a atuação profissional é embasada nos processos de formação profissional, sejam atividades práticas ou teóricas, o presente artigo destina-se a identificar a percepção dos estudantes universitários da área de saúde quanto à obesidade. O objetivo é contribuir com questionamentos e reflexões, assim como identificar a necessidade de possíveis alterações neste processo de formação.

\section{MÉTODOS}

A pesquisa foi aplicada em universitários dos cursos de graduação em saúde no Campus da Faculdade de Medicina do ABC (FMABC), localizada em Santo André, São Paulo, Brasil. 
Chegou-se a um total de 39 universitários, sendo 32 do sexo feminino e 7 do masculino, entre 17 e 27 anos, estudantes dos cursos de Medicina, Fisioterapia, Nutrição e Terapia Ocupacional.

Os estudantes foram convidados para participarem da pesquisa. Após as aulas presenciais, foram esclarecidos sobre o objetivo e a finalidade da pesquisa, assim como autorizaram o uso de suas respostas por meio de assinatura ao termo de consentimento, conforme orientação do Comitê de Ética em Pesquisa. A pesquisa foi aprovada sob protocolo número 126/2009.

A investigação foi realizada em duas etapas:

- apresentação de nove ilustrações, como visto na Figura 1, uma de cada vez, por meio de projetor de multimídias, seguida da anotação das percepções e dos sentimentos despertados após a visualização de cada figura em uma filipeta de papel sem identificação (que foi distribuída antes da apresentação). Elas foram escolhidas aleatoriamente, em revistas diversas, e estavam relacionadas à alimentação, ao peso, ao grupo social e à superação das limitações físicas;

- a segunda etapa da pesquisa foi realizada por meio de questionário, composto por três perguntas formuladas no software Qualiquantisoft, v1.3c (Sales \& Paschoal Informática): a) É difícil não ser um pouco preconceituoso em relação a pessoas obesas, não é? Como fica isso para você? Fale um pouco sobre isso; b) Obesidade é sempre uma doença? Fale um pouco sobre isso; c) Obesidade é diferente para o homem e para a mulher? Qual a sua opinião sobre isso?

A análise dos resultados foi feita por meio de abordagem qualitativa e quantitativa, com o auxílio do software Qualiquantisoft, que utiliza a técnica do discurso do sujeito coletivo (DSC) ${ }^{17}$. Esta é um recurso metodológico que permite a realização de pesquisas de resgate das opiniões coletivas. É uma proposta de coleta, organização, tabulação e análise dos dados qualitativos de discurso do sujeito, por meio de meios verbais ou escritos, tanto individuais quanto coletivos.

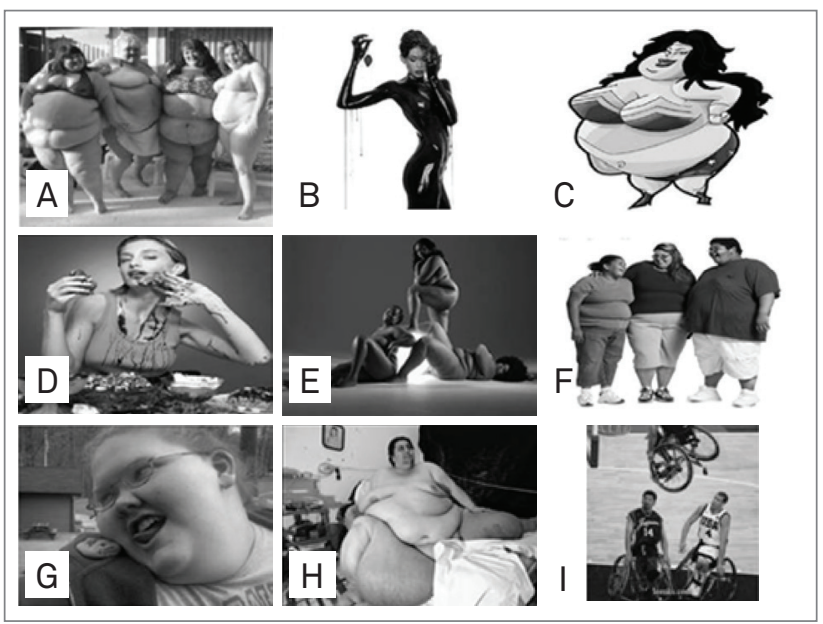

Figura 1: Figuras apresentadas aos estudantes como disparadoras da primeira etapa da pesquisa
O DSC é composto por quatro etapas: seleção das expressões-chave, isto é, de trechos que melhor descrevam o conteúdo explanado; formulação das ideias centrais, compostas por respostas ou conjuntos delas que apresentam sentido familiar ou complementar; após a formulação das ideias centrais, pode-se obter as ancoragens, que descrevem as ideologias, os valores, as crenças, presentes no material das respostas individuais e/ou agrupadas, sob a forma de afirmações genéricas destinadas a enquadrar situações particulares. Finalmente, temos a quarta etapa, na qual serão realizados os DSCs propriamente ditos, que por consequência reúnem as expressões-chave presentes nos depoimentos, com ideias centrais e/ou ancoragens de sentido familiar ou complementar.

Tanto as perguntas como as imagens se destinaram a avaliar percepções, sentimentos e pensamentos dos participantes. Todas as respostas, tanto aquelas obtidas após a apresentação das figuras quanto das questões, foram agrupadas conforme as ideias centrais mostradas, a fim de facilitar a classificação, a tabulação e a análise dos dados.

\section{RESULTADOS E DISCUSSÃO}

Os dados apontam maior frequência de ideia ou postura de rejeição frente às figuras de pessoas com sobrepeso/obesidade em situações sociais ou cotidianas, assim como sugerem associações frequentes do sobrepeso/obesidade a termos e expressões pejorativas. Em contrapartida, as imagens de pessoas eutróficas remeteram a associações de saúde e sensualidade.

As principais informações encontradas serão apresentadas a seguir, destacando-se aquelas de maior relevância ao objetivo do estudo. Serão declarados, inicialmente, os resultados da primeira etapa da pesquisa.

Diante da Figura 1A, 27,91\% dos pesquisados manifestaram uma postura de rejeição aos padrões estéticos, respondendo: "não quero ficar assim"; "exposição ao ridículo"; "o excesso é feio e bizarro" e "nojo". Porém, 25,58\% indicaram aceitação, com expressões como: "estereótipos não necessariamente fazem ou deixam de fazer as pessoas felizes"; "apesar de estar fora dos padrões da sociedade, assumir o que ele é, é bem mais legal" e "ser feliz com os próprios defeitos". Um total de 9,30\% demonstrou uma postura crítica em relação ao amor, ao respeito pelo corpo e à autoimagem, referindo: "exposição ao ridículo como tentativa de aceitação"; "eu acho que elas são gordas, mas não se enxergam como tal" e "não se importam com a aparência”. Apesar da frequência de postura de aceitação e rejeição ser próxima, é importante destacar que, mesmo nas expressões de aceitação, é possível observar palavras que remetem à marginalização da obesidade.

Frente à Figura 1B, 32,56\% dos pesquisados relacionaram a modelo à aparência, com afirmações como: "ditadura da moda" e "culto ao corpo perfeito". Já 20,93\% associaram à sedução e sensualidade, por meio de frases como: "tentativa de ser sexy"; 
"sensualidade forçada" e "sedução e tentação". Apenas 9,30\% citaram que a figura tinha relação a aspectos de sujeira, com expressões como: "que lambança" e "meleca". Portanto, no total, 53,49\% dos participantes remeteram-se a um padrão de beleza que valoriza o corpo e a sensualidade.

As reações à Figura $1 \mathrm{C}$ enfatizam uma postura de rejeição/ crítica, atingindo 44,19\% dos pesquisados, evidenciada pelos argumentos: "quebra de padrão de beleza"; "falta de critérios" e "pessoas inadequadas chamam atenção". Enquanto 25,58\% demostraram aceitação, por meio das expressões: "essa imagem representa força de vontade"; "se sentir bonita apesar do peso"; "não estar nos padrões de beleza também pode ser sexy" e "para ser mulher maravilha, tem que ser você mesma". Enquanto 13,95\% adotaram como ideia central a contradição e incoerência, sob as alegações: "representa uma contradição, pois as características não são compatíveis com as de um modelo de heroína"; "obesidade contra a beleza" e "sátira e cômico".

Frente à Figura 1D, 34,88\% dos participantes pensaram em prazer sem culpa, com expressões como: "vontade de comer sem culpa" e "fazer o que tem vontade, sem limites". Um porcentual de 32,56 pensou em compulsão, gula e abuso: "gula desnecessária", "comer sem pensar nas consequências" e "garota com compulsão alimentar". Por outro lado, 6,98\% associaram a figura à sensação de sujeira e nojo: "muita sujeira só para comer doces" e "essa imagem me remete a abuso". Somando-se, 67,44\% deles relacionaram ideias de falta de limite ou culpa à Figura 1D.

Quanto à Figura 1E, as ideias centrais apresentadas por 44,19\% dos sujeitos foram de aceitação, com falas como: "gordinhas também se sentem sexy" e "a beleza está dentro de você, mas não em seu corpo". Enquanto 37,21\% tiveram ideias de rejeição/crítica, por meio de expressões: "sensualidade fora dos padrões aceitos atualmente"; "imagem ridícula" e "gordo não é sexy". Apenas 9,30\% mencionaram aspectos de saúde: "sensual, mas não é saudável" e "esforço para ser sensual, mas doente".

A Figura $1 \mathrm{~F}$ remeteu $72,09 \%$ dos participantes à ideia de família e/ou amigos unidos, dos quais $16,28 \%$ associaram a obesidade a hábitos e genética não saudável, afirmando: "família que come unida permanece unida"; "tal pai, tal filho"; "união e amor, mas sem cuidado" e "obesidade é realmente genética". Ideias de aceitação e rejeição/crítica foram relatadas igualmente por $13,95 \%$ dos indivíduos.

As ideias centrais mostradas por $39,53 \%$ dos participantes, diante da Figura 1G, foram de doença, gula, bullying e compulsão, expressas por: "a obesidade atrapalha a vida das pessoas"; "a importância de conhecer o limite do que é saudável"; "por isso que é gorda, só pensa em comida"; "comer a qualquer custo"; "busca compulsória por comida desagradável"; "algo triste, feio, doente" e "depois não sabe por que sofre bullying". Já 30,23\% apresentaram rejeição/critica, por meio de falas como: "por que ele tira essa foto?" e "seria melhor não conseguir comer”. Dessa forma, 69,76\% associaram a Figura $1 \mathrm{G}$ a aspectos negativos da vida psicossocial das pessoas. Apenas 9,30\% relacionaram-na à busca pela felicidade: "força de vontade para alcançar o que se quer" e "não importa o motivo da felicidade".

A Figura $1 \mathrm{H}$ suscitou ideias de infelicidade, sofrimento, doença ou prejuízo, distribuídas em: 37,21\% associaram especificamente à infelicidade, ao infortúnio, à baixa estima, ao sofrimento e à imagem deplorável; 32,56\% à morbidade, ao tratamento, à necessidade de mudança; e 11,63\% consideraram um prejuízo na vida social. As principais expressões foram: "como alguém se permite chegar a esse ponto?"; "ele precisa de ajuda, de tratamento"; "degradação de um ser humano"; "falta de saúde, em todos os aspectos"; "impedido de se relacionar com as pessoas"; "não é possível viver assim" e "só consigo ver tristeza".

Já a Figura 1I remeteu 95,35\% dos participantes a ideias de superação, expansão de limites, lição de vida e coragem, realização de sonhos e objetivos, vitória, reportando com as seguintes expressões: "você é quem define seus próprios limites"; "superação é tudo na vida" e "exemplo de autoaceitação e coragem".

Os resultados obtidos na segunda etapa da pesquisa serão expostos subdivididos pelas questões formuladas.

Com relação à primeira questão, sobre o preconceito com os obesos, os principais dados indicam que $33,33 \%$ dos participantes não se consideram preconceituosos quanto à obesidade; em contrapartida, $25,64 \%$ afirmaram que toda a sociedade é preconceituosa, apesar de não assumir isso e 23,08\% declararam serem.

Não sou preconceituoso em relação a isso, [...] tento deixar os estereótipos de lado.

[...] o preconceito existe é verdade, e não é pouco, principalmente na nossa atual sociedade, que impõe um padrão de beleza [...].

No fundo, todos têm preconceito com as pessoas gordas, mas alguns escondem isso. A obesidade nos incomoda, [...] porque a sociedade não está pronta para aceitar as diferenças [...]. O mundo não é dos gordinhos!

Quando questionados sobre a obesidade como doença, 71,49\% consideram-na, sendo que destes $33,33 \%$ indicaram a necessidade de se levar em conta fatores psicossociais associados; $12,82 \%$ indicaram que a obesidade pode desencadear outras doenças; $12,82 \%$ relataram que a obesidade é causa de uma vida não saudável, apesar de muitas pessoas não entenderem isso; 7,69\% afirmaram que ela não é uma doença até começar a atrapalhar a vida do sujeito; e 15,38\% mostraram que tal condição pode ser sedentarismo, relaxo ou descuido da pessoa, associando a esta a responsabilidade pela obesidade.

A respeito da diferença entre a obesidade em homens e mulheres, $77,10 \%$ consideraram que há diferenças significativas, sendo que $64,10 \%$ atribuíram ela à cobrança social em relação ao corpo de ambos, 7,69\% relacionaram à influência hormonal e $5,13 \%$ não justificaram a resposta. Somente $20,34 \%$ consideraram que não há diferenças. 
Retomando os dados apresentados, cabe destacar que aproximadamente $80 \%$ dos sujeitos da pesquisa consideram a obesidade como uma doença, em contraposição aos 15\% que não a percebe como uma enfermidade. Apesar disso, ideias e postura de rejeição/crítica foram apresentadas com maior frequência pelos participantes, assim como a maioria dos sujeitos da pesquisa afirmou a existência de preconceito contra obesos.

Dessa forma, pode-se considerar que há distorções relativas à percepção da obesidade enquanto doença, sua etiologia, agravos e comorbidades, assim como quanto aos processos de prevenção, recuperação e reabilitação de pacientes com sobrepeso/obesidade.

A pesquisa evidencia que mesmo estudantes da área de saúde têm dificuldade de desvencilhar-se do preconceito existente na sociedade em geral, considerando com maior frequência a aparência e a necessidade de mudanças para atender às imposições estéticas da atualidade e não aos aspectos de bem-estar, autoestima, qualidade de vida ou biopsicossociais de saúde/doença.

Os resultados indicam dificuldade em distanciar-se de preconcepções estético-corporais, do culto ao corpo, da modelação corporal e das cirurgias, além da perda de peso e prática de exercícios. Pode-se compreender que o discurso dos universitários está atrelado ao padrão de beleza atual, reproduzindo-o, por vezes.

Assim como descrito em pesquisas anteriores ${ }^{16}$, os participantes desta investigação demonstraram ideias associadas à responsabilização do indivíduo pela obesidade, principalmente pela transgressão de regras e limites e/ou por não terem comportamentos adequados que levariam à perda de peso.

A percepção da obesidade parece restrita a tal responsabilização dos indivíduos obesos, desconsiderando-se as dimensões históricas, psicológicas e sociais, como valores culturais, posição socioeconômica, histórico de vida, fatores genéticos ou hereditários.

O discurso dos sujeitos da pesquisa evidencia, ainda, um forte sentimento desfavorável e de recusa quanto ao corpo obeso, mencionando expressões que remetem a escondê-lo ou encobri-lo, que não merece ser exposto ou visto aos olhos dos demais. Cabe destacar ainda que a apresentação das imagens de pessoas obesas em diversas situações não despertou ideias de superação da obesidade, como aconteceu diante da Figura 1.

É importante considerar o impacto de percepções, ideias, representações, sensações, crenças internas e sentimentos daqueles que se dedicam ao atendimento e tratamento de pacientes obesos. A presença de ideias predominantes de rejeição e crítica pode influenciar o tratamento, comprometendo-o, já que não favorece a empatia, a compreensão, o acolhimento e/ou o apoio e outros subsídios necessários para uma abordagem adequada da obesidade como patologia multifatorial ${ }^{18}$. O indivíduo obeso, muitas vezes, já conserva uma autopercepção corporal distorcida e sentimentos negativos a respeito de si, requerendo a assistência de profissionais solidamente preparados para enfrentar tais questões.

A construção da imagem corporal é fortemente influenciada pelo olhar dos outros e impacta diretamente em diversos contextos da vida, refletindo nas atitudes, no comportamento, nos pensamentos, nas reações às situações da vida e nas relações profissionais, sociais, pessoais e afetivas ${ }^{19}$.

Portanto, é primordial sensibilizar, orientar e construir vínculos com o paciente, os familiares e os profissionais envolvidos. A perda de peso para o indivíduo obeso não deve ser o fator principal do processo, mas apenas um deles, uma vez que outros estão envolvidos, tal como a reconstrução da imagem corporal, com a adequada percepção psicológica de $\mathrm{si}^{20}$.

Apesar dos sujeitos deste trabalho entenderem que a doença deve ser tratada, os resultados indicam a necessidade de abordar aspectos psíquicos e considerar questões pessoais e internas destes, a fim de que possam contribuir com excelência para os tratamentos necessários ${ }^{20}$.

No intuito de aprimorar o tratamento de pacientes com sobrepeso/obesidade, sugere-se a realização de outras pesquisas na área, assim como a inclusão de atividades de sensibilização e orientação nos cursos de graduação em saúde.

Como considerações finais, primeiramente cabe destacar que esta pesquisa foi impulsionada pela atuação das autoras em um ambulatório didático, em que a prática clínica se imbrica com a participação no processo de formação prática dos estudantes de diversos cursos de graduação e pós-graduação em saúde.

A fim de viabilizar a pesquisa e minimizar os vieses de nossa própria atuação nesse ambulatório didático, os alunos convidados a participarem da pesquisa não haviam participado de atividades em tal local. Foi sugerida aqui uma nova pesquisa, com o objetivo de verificar a percepção acerca da obesidade dos alunos de cursos de graduação ou pós-graduação em saúde, após as atividades práticas em um ambulatório multidisciplinar.

É importante destacar os limites deste trabalho, que teve caráter inicial e exploratório. A principal fragilidade desta pesquisa relaciona-se à escolha das figuras e formulação das questões, que podem ter suscitado respostas tendenciosas. Portanto, sugere-se a realização de novas investigações que incluam outras imagens e questões e que possam abranger outras variáveis. Além disso, atingiu-se uma quantidade limitada de sujeitos e cursos de graduação em saúde.

Dessa forma, compreende-se que a pesquisa pode funcionar como disparadora de outras ou de uma mais ampla e melhor delimitada.

Apesar disso, foram revelados dados importantes que podem servir como um alerta de que a questão da obesidade precisa ser mais bem abordada nos cursos de formação da área de saúde, a fim de se reduzir os efeitos negativos dessa rejeição/crítica no decorrer do tratamento de tais pacientes. 


\section{REFERÊNCIAS}

1. World Health Organization. Obesity: preventing and managing the global epidemic. Geneva: WHO; 2000.

2. Pinheiro AR, Freitas SF, Corso AC. Uma abordagem epidemiológica da obesidade. Rev Nutr. 2004;17(4):523-33. http://dx.doi.org/10.1590/S1415-52732004000400012

3. Fisberg M. Obesidade na infância e na adolescência. Porto Alegre: Fundação Byk; 1995.

4. Boog MC. Educação nutricional em serviços Públicos de Saúde. Cad Saúde Pública. 1999:15(Suppl 2):S139-47. http://dx.doi.org/10.1590/S0102-311X1999000600014

5. Assumpção JR, Francisco B, Kaczynski E. Tratado de psiquiatria da infância e adolescência. São Paulo: Atheneu; 2003.

6. Almeida GA, Loureiro SR, Santos JE. A imagem corporal de mulheres morbidamente obesas avaliadas através do desenho da figura humana. Rev Psicol Reflex Crít. 2002;15(2):283-92. http://dx.doi.org/10.1590/S0102-79722002000200006

7. Nunes MA, Appolinario JC, Galvão AL, Coutinho W. Transtornos alimentares e obesidade. São Paulo: Artmed: 2006.

8. Instituto Brasileiro de Geografia e Estatística (IBGE). Pesquisa de orçamentos familiares 2008-2009: análise do consumo alimentar pessoal no Brasil. Rio de Janeiro; 2011.

9. Serra GM, Santos EM. Saúde e mídia na construção da obesidade e do corpo perfeito. Cienc Saúde Coletiva. 2003;8(3):691-701. http://dx.doi.org/10.1590/S1413-81232003000300004

10. Serra GMA. Saúde e nutrição na adolescência: o discurso sobre dietas na revista capricho. Dissertação (Mestrado) - Fundação Oswaldo Cruz, Escola Nacional de Saúde Pública, Rio de Janeiro, 2001, p. 17-27.

11. Puhl RM, Heuer CA. The stigma of obesity: a review and update. Obesity. 2009;17(5):941-64. http://dx.doi.org/10.1038/oby.2008.636
12. Segal A, Fandino J. Indicações e contra-indicações para realização das operações bariátricas. Rev Bras Psiquiatr 2002;24(Suppl 3):68-72.

http://dx.doi.org/10.1590/S1516-44462002000700015

13. Ferrarini MG, Dias TS, Silva KZ, Martins CS. Auto-imagem corpora de adolescentes atendidos em um programa multidisciplinar de assistência ao adolescente obeso. Rev Bras Saúde Materno Infantil. 2005;5(1):27-33.

http://dx.doi.org/10.1590/S1519-38292005000100004

14. Zottis C, Lanbronici LM. O corpo obeso e a percepção de si. Cogitare Enferm. 2002;7(2):21-9.

http://dx.doi.org/10.5380\%2Fce.v7i2.1665

15. Vilhena J, Novaes JV, Rocha L. Comendo, comendo e não se satisfazendo: apenas uma questão cirúrgica? Obesidade mórbida e o culto ao corpo na sociedade contemporânea. Rev Mal Estar Subjet. 2008;8(2):379-406.

16. Laurini KC. Obesidade feminina: olhares sobre o corpo Piracicaba: Unimep; 2009

17. Lefèvre F, Lefèvre AM, Teixeira JJ. O discurso do sujeito coletivo: uma nova abordagem metodológica em pesquisa qualitativa Caxias do Sul: EDUCS; 2000.

18. Repetto G. Histórico da obesidade. In: Halpern A, Godoy MA, Suplicy HL, Mancini C, Zanella MT. Obesidade. São Paulo: Lemos; 1998.

19. Veggi AB, Lopes CS, Faerstein E, Sichieri R. Índice de massa corporal, percepção do peso corporal e transtornos mentais comuns entre funcionários de uma universidade no Rio de Janeiro. Rev Bras Psiquiatr. 2004;26(4):242-7. http://dx.doi.org/10.1590/S1516-44462004000400007

20. Schilder P. A imagem do corpo. São Paulo: Martins Fontes; 1994. 